
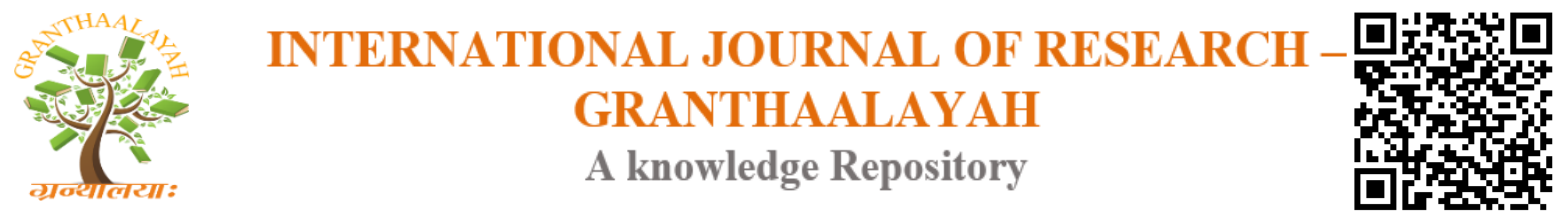

Science

\title{
ASSESSMENT OF NORMAL LIVER PORTAL VEIN AND COMMON BILE DUCT USING DOPPLER ULTRASONOGRAPHY
}

\author{
Ashraf Mustafa ${ }^{1}$, Mohamad Elfadi ${ }^{1}$ Mohamed Gar Elnabi ${ }^{2}$, Mona Ahmed ${ }^{3}$, \\ Abdelmoneim Sulieman 1, *2 \\ ${ }^{1}$ College of Medical Radiologic Science, Sudan University of Science and Technology, P.O.Box \\ 1908, Khartoum 11111, Sudan \\ ${ }^{2}$ Prince Sattam Bin Abdulaziz Universities, College of Applied Medical Sciences, Alkharj, \\ Saudi Arabia
}

\begin{abstract}
Ultrasound imaging plays a vital role in the evaluation of many tissue or organs disorder. The objective of this study was to evaluate normal liver portal vein (PV) and common bile duct (CBD) using Doppler Ultrasonography (Duplex). Two ultrasound machines equipped with linear and curvilinear probes used in this study (Toshiba and ALOKA, Japan) during the period from 1st April 2016 to 30th July 2017. A total of 300 (48\% males; 52\% females) healthy subjects were participated in this study with age range from 16-22 years old. The mean intrahepatic portal vein velocity is between $(14.98 \pm 1.92) \mathrm{cm} / \mathrm{s}$; and the diameter range is $(9.76 \pm 2.09)$. In frequency distribution of the extrahepatic portal vein velocity values, the majority is in the range $(14-15 \mathrm{~cm} / \mathrm{s})$ which is equal to the half of the examined subjects. The extra hepatic PV versus intra hepatic portal vein velocities was found to be more in normal liver, where the velocity increased by $0.31 \mathrm{~cm} / \mathrm{s}$ inside the liver per each $\mathrm{cm} / \mathrm{s}$ of the extrahepatic for the normal liver.
\end{abstract}

Keywords: Liver Doppler; Portal Vein; Common Bile Duct (CBD); Ultrasound Imaging; Liver.

Cite This Article: Ashraf Mustafa, Mohamad Elfadi Mohamed Gar Elnabi, Mona Ahmed, and Abdelmoneim Sulieman (2018). "ASSESSMENT OF NORMAL LIVER PORTAL VEIN AND COMMON BILE DUCT USING DOPPLER ULTRASONOGRAPHY." International Journal of Research - Granthaalayah, 6(11), 384-390. 10.29121/granthaalayah.v6.111.2018.1141.

\section{Introduction}

Vascular anatomy and normal flow profile: Duplex Doppler Ultrasonography of the liver plays an important role in the evaluation of the liver1,2. This noninvasive, ionizing radiation-free, portable technique is not only able to quickly assess patency of the hepatic vasculature but it is also able to provide additional parameters such as resistivity and direction of flow. Other articles have reviewed imaging evaluation of the liver vasculature 3,4. The portal vein is formed by the confluence of the splenic and superior mesenteric veins. It supplies around $70 \%$ of incoming blood volume to the liver. It is accompanied by the hepatic artery and common bile duct which to get her 
make up the portal triad (Figure 1). The triad has echogenic margins as it enters the liver, due to the intrahepatic extension of Glisson's capsule and presence of perivascular fat. After forming the right and left branches, these vessels progressively branch to supply the liver segments.

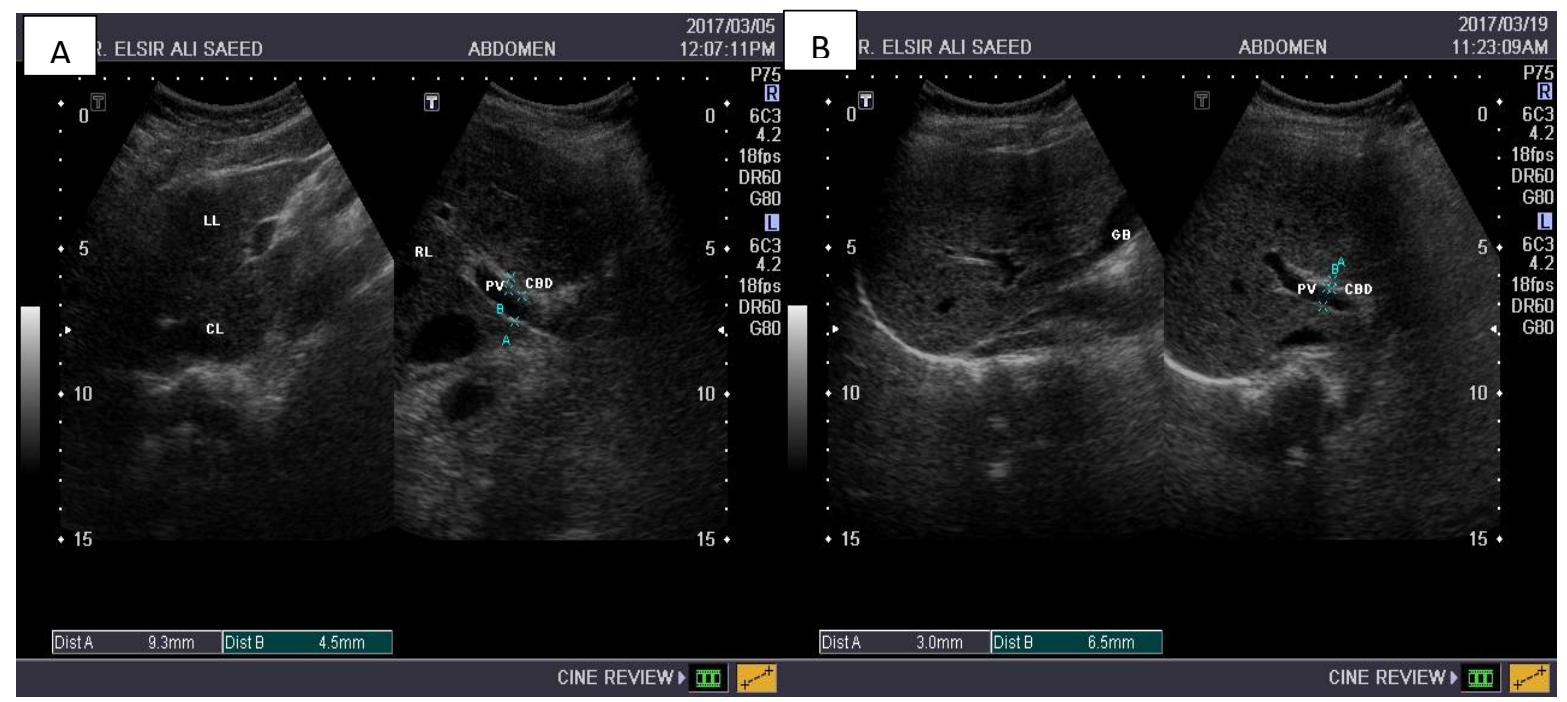

Figure A\&B 1: intrahepatic portal vein \& the common bile duct (CBD), in normal individual.

The biliary tree is the sum of tubes that transport bile which is secreted from the hepatocytes, stored in the gallbladder and at the end throughout the common bile duct the contents poured in the second part of the duodenum together with the pancreatic duct at the ampulla of Vator. The right and left bile ducts unite to form the common hepatic duct which join the cystic duct from the gallbladder to form the CBD. The portal vein Doppler flow profile in a fasting patient has a relatively constant velocity of approximately $18 \mathrm{~cm} / \mathrm{s}$ towards the liver (hepatopetal) (Figure 2). The flow velocity is uniform because cardiac pulsation is damped by the splenic parenchyma and the capillaries of the gut at one end of the portal system and by the liver sinusoids at the end of the portal venous system. General examination of liver parenchyma and abdomen is a must then color and spectral Doppler assessment of the portal vein, superior mesenteric and splenic veins, together with main intrahepatic portal branches. Color and spectral Doppler assessment of the hepatic artery from the coeliac axis to the porta hepatis, together with main intrahepatic branches Color and spectral Doppler assessment of the main hepatic veins and the upper inferior vena cava finally, assessment of flow of the feeding vessels to any observed intrahepatic mass or abnormality. Previous studies show normal values of the portal vein diameter ranges between $(6-13 \mathrm{~mm})$; no values are registered for the intrahepatic length (future study); the normal velocity was up to $20 \mathrm{~cm} / \mathrm{s}$; of hepatopetal flow $^{1-2}$.

The purpose of our study was to identify specific Doppler criteria for portal vein and outflow vein (hepatic veins and inferior vena cava), as well as the neighboring channel (the CBD) in normal liver. so as to relate this in the future with the livers of patients that had liver failure or those which are planned for or did (underwent) transplantation. 

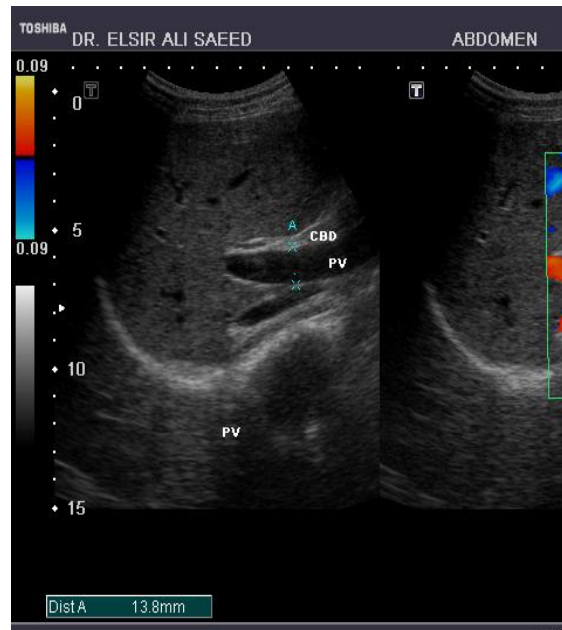

(T)
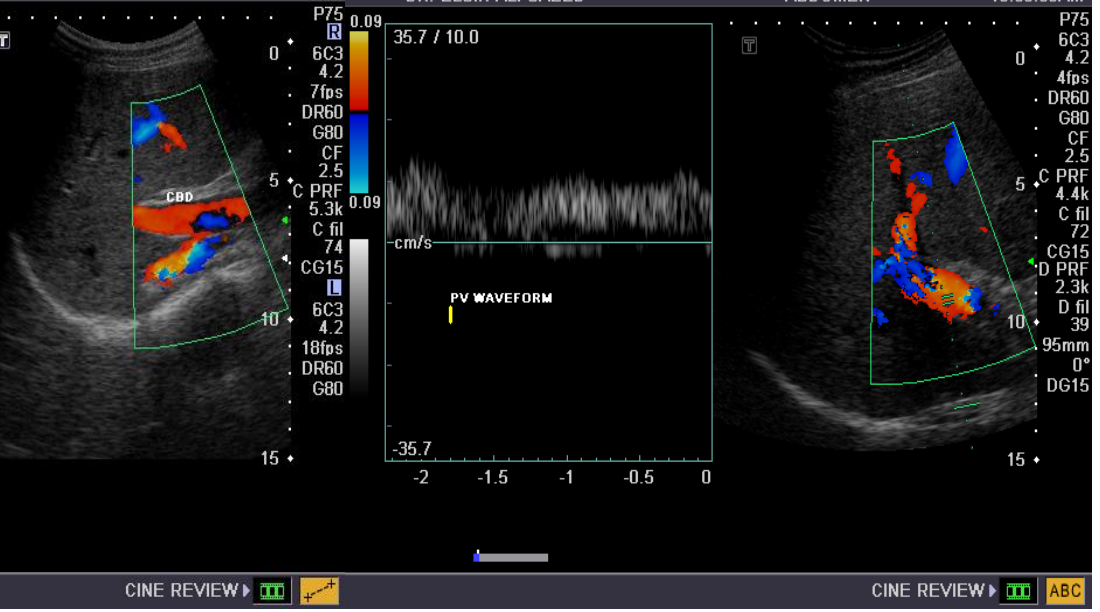

Figure 2: color flow \& spectral trace of normal portal vein.

\section{Materials and Methods}

Methods of data collection= using the data sheet; both transverse and longitudinal technique in putting the transducer in the mid clavicular line and coronal oblique in the anterior axillary line intercostally for PV, CBD \& HA; mid-line sub costal oblique to trace the hepatic veins; mid line transverse for the HA (seagull sign below the pancreas).

Sample size: the data had been collected from 300 subjects (48 M; 52\% F). All study population are bachelor students in faculty of medicine National Ribat University. Ethical approval has been granted from the hospital and the department of GIT bleeding and liver disease. In addition, consent from the patients was signed and oral agreement after they understand what will be done in the study. This did not include or disclose any [ID] information concerning the patient.

Equipment: Two ultrasound machines of complete capabilities (Toshiba and ALOKA) equipped with two

Data Analysis: The data was collected from the three hundred expected normal student of faculty of medicine in Al Rabat University during the period from $1^{\text {st }}$ April 2016 to $30^{\text {th }}$ July 2017, and it is analyzed using the Statistical Package for Social Science - SPSS version 20.0.

\section{Results and Discussions}

The portal vein normal velocity was found to be less than $20.0 \mathrm{~cm} / \mathrm{s}$ in normal young Sudanese students. The majority ranges between $(8-18 \mathrm{~cm} / \mathrm{s})$ and there is strong relationship between the site (intra or extra hepatic) and the size in both dimensions (caliber and length); the common bile duct is not more than $6.0 \mathrm{~mm}$ the majority ranges between $(3-6 \mathrm{~mm})$. Doppler US is the main initial imaging modality for the evaluation and assessment of liver vasculature patency as well as direction of flow due to its easy availability and high sensitivity in the detection of vascular as well as biliary complications. 
In Table (1), which shows the PV velocity both inside and outside the liver plus the internal caliber, it reveals that the mean intrahepatic portal vein velocity is between $(14.98 \pm 1.92) \mathrm{cm} / \mathrm{s}$; and the diameter range is $(9.76 \pm 2.09)$. In frequency distribution of the extrahepatic portal vein velocity values, the majority is in the range $(14-15 \mathrm{~cm} / \mathrm{s})$ which is equal to the half of the tested subjects. On the other hand, the intra - Hepatic PV velocity values ranging between $(15-16 \mathrm{~cm} / \mathrm{s})$ represents the higher frequency in normal livers $(36 \%)$. Figures $(1,2,3)$ show the intrahepatic portal vein caliber; the higher frequency of the calibers of the intrahepatic portal vein i.e. The majority is in the group $(9-10 \mathrm{~mm})$ with a $46.0 \%$, near the half of the tested subjects.

Figures $(3,4,5)$ show a scatter plot of hepatic PV velocity versus the caliber of the portal vein where the velocity increases directly by $0.19 \mathrm{~cm} / \mathrm{s} / \mathrm{mm}$ of caliber for normal liver; this increment is given by the function ( $y=0.1864 x+13.159$, when $\left.\mathrm{R}^{2}=0.412\right)$, this mean that there is a significant relationship between the velocity and the caliber. However, it also show the scatter plot of extra hepatic PV velocity versus portal vein caliber ; in this case the story is different; where the velocity decreases by $0.037 \mathrm{~cm} / \mathrm{s} / \mathrm{mm}$ of caliber for normal liver given by the first degree equation $(\mathrm{y}=$ $0.0366 \mathrm{x}+13.973)$.

The extra hepatic PV versus intra hepatic portal vein velocities was found to be more in normal liver, where the velocity increased by $0.31 \mathrm{~cm} / \mathrm{s}$ inside the liver per each $\mathrm{cm} / \mathrm{s}$ of the extrahepatic for the normal liver. This significant relation reflects that the caliber is anatomically decreased inside, see scatter plot, given by the function $(y=0.3108 x+8.9607)$.

CT is complementary to US and is often used as a problem-solving modality, being reserved for second-line investigation when US findings are indeterminate or inconclusive. Cholescintigraphy remains the most sensitive noninvasive modality for the detection of bile leak or sludge after ultrasonography. MR cholangiopancreatography is very useful in the evaluation of bile duct dilatation and obstruction. Imaging is useful for the detection of early and late complications, as well as for long-term follow-up to assess the portal vein. An understanding of potential pathological vascular liver complications and of the strengths and weaknesses of each imaging modality will aid in early diagnosis and promote timely therapy. EUS is an excellent imaging modality for evaluation of vascular anatomy. Previous studies showed that ultrasound is noninvasive imaging technique and useful for assessment of the liver anatomy ${ }^{3-10}$. Current ultrasound machines provide high resolution images providing accurate measurement for the liver anatomy.

Table 1: The PV velocity both inside and outside the liver plus internal caliber

\begin{tabular}{|l|l|l|l|}
\hline Liver & Mean \pm SD & $\min$ & $\max$ \\
\hline Extra hepatic PV & $13.62 \pm 1.3$ & 10 & 17 \\
\hline Velocity of PV & $14.98 \pm 1.92$ & 9.5 & 19 \\
\hline Portal vein caliber & $9.76 \pm 2.09$ & 3.3 & 13.8 \\
\hline
\end{tabular}




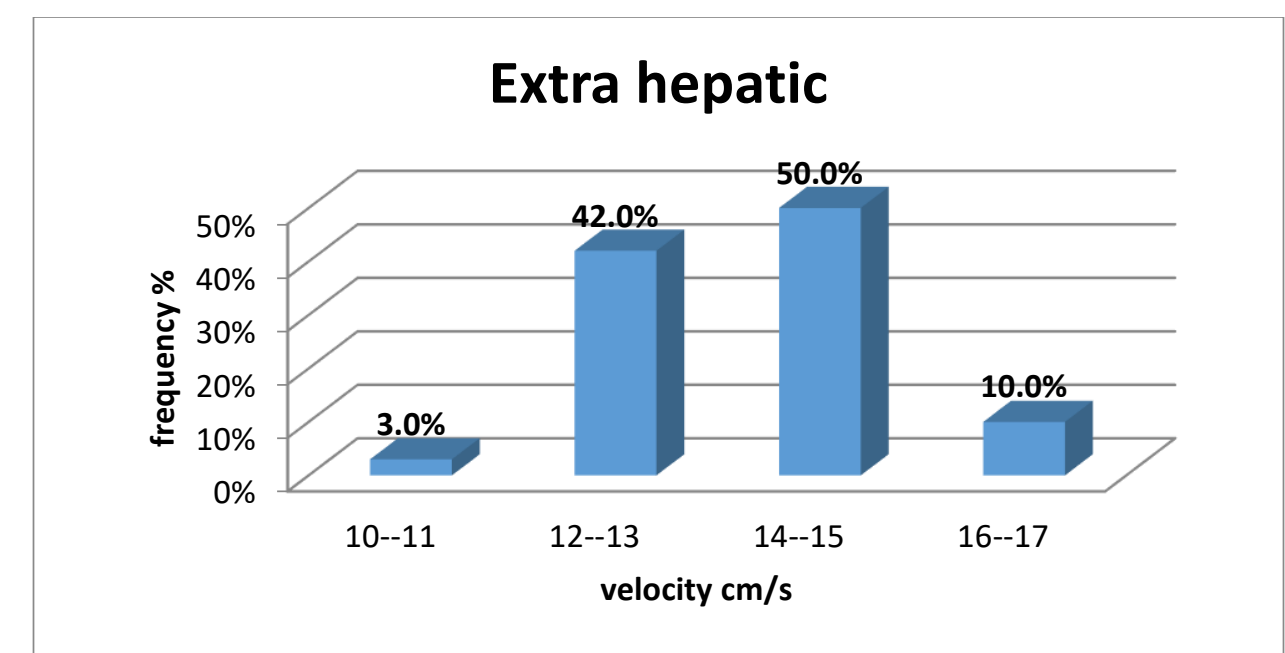

Figure 3: The frequency of extrahepatic portal vein velocity values, the majority is the range (14$15 \mathrm{~cm} / \mathrm{s}$ ) which is equal to the half of the tested subjects.

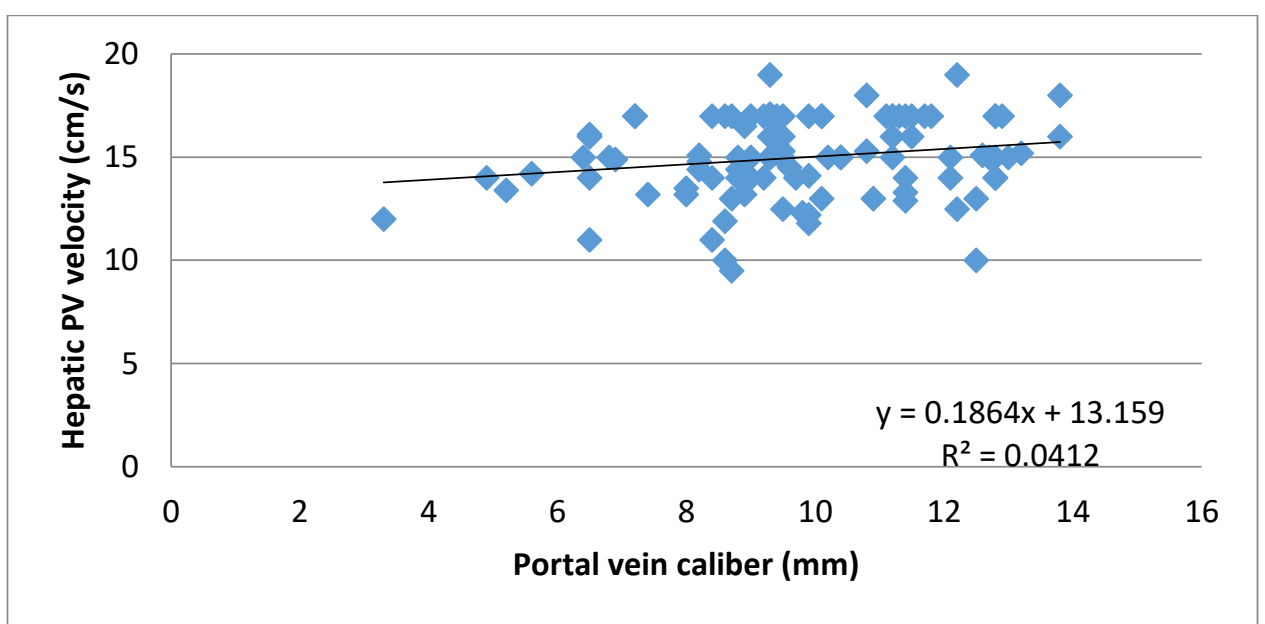

Figure 4: scatter plot of intra - hepatic PV velocity versus the caliber of the portal vein where the velocity increases directly by $0.19 \mathrm{~cm} / \mathrm{s} / \mathrm{mm}$ of caliber for normal liver.

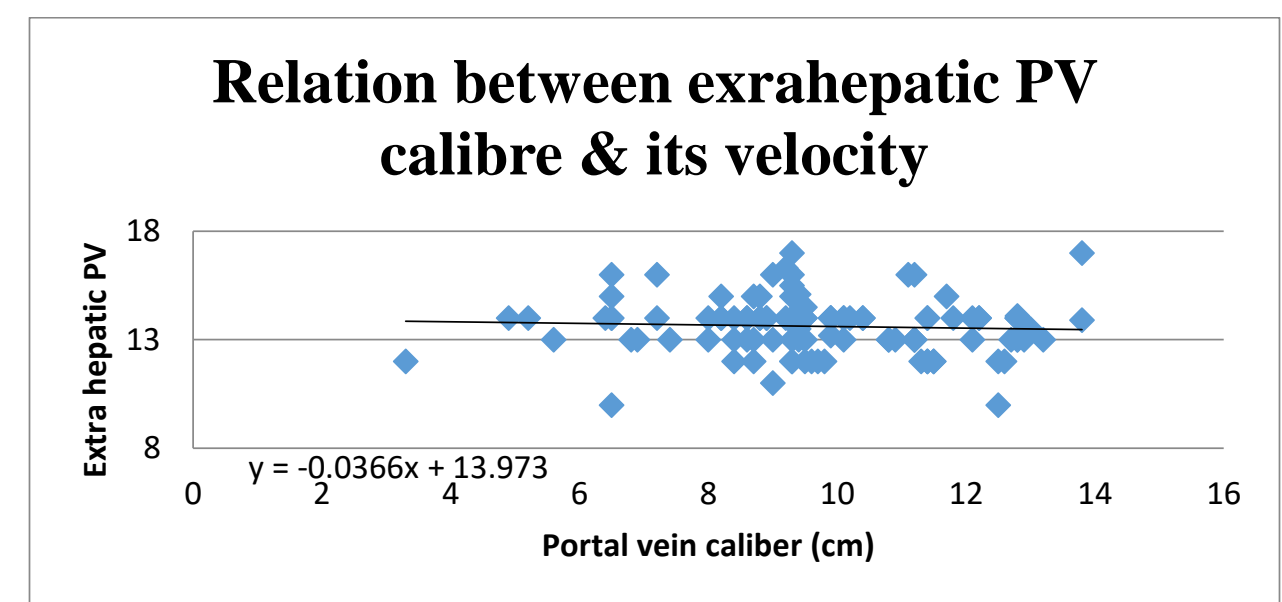

Figure 5: scatter plot of extra hepatic PV velocity versus portal vein caliber where the velocity decreases by $0.037 \mathrm{~cm} / \mathrm{s} / \mathrm{mm}$ of caliber for normal liver. 


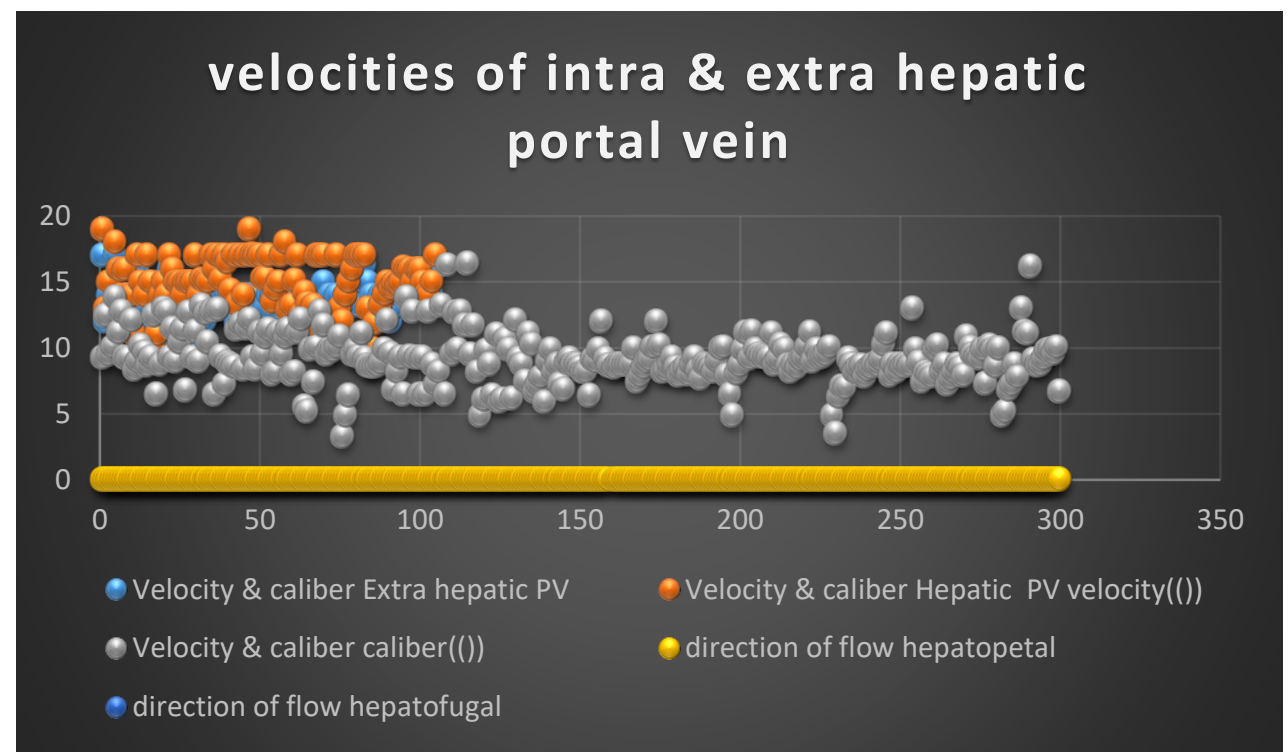

Figure 6: Combined mixed colored scatter plot of both extra \& intra hepatic PV velocity together with their calibers and direction of flow.

\section{Conclusions and Recommendations}

Multiple complications can be observed after liver transplantation. US is the main initial imaging modality for the evaluation of liver transplant dysfunction due to its easy availability and high sensitivity in the detection of vascular as well as biliary complications. CT is complementary to US and is often used as a problem-solving modality, being reserved for second-line investigation when US findings are indeterminate or inconclusive.

\section{References}

[1] B. Deepanraj, P. Lawrence, G. Sankaranarayanan, Theoretical analysis of gas turbine blade by Finite element method, Scientific World, Vol. 9, No. 9, July 2011, 29-33.

Abu-Yousef MM. (Normal and respiratory variations of the hepatic and portal venous duplex Doppler waveforms with simultaneous electrocardiographic correlation. J Ultrasound Med 11(6) 1992, 263-8

[2] Goyal N, Jain N, Rachapalli V, et al. Non-invasive evaluation of liver cirrhosis using ultrasound. Clinical Radiol 64 (11):2009. 1056-66.

[3] Gorg C, Riera-Knorrenschild J, Dietrich J. Colour Doppler ultrasound flow patterns in the portal venous system. Br J Radiol .75(899):2002.919-929.

[4] Gallix BP, Taourel P, Dauzat M, et al. Flow pulsatility in the portal venous system: a study of Doppler sonography in healthy adults. AJR Am J Roentgenol .169 (1):1997. 141-4.

[5] Wood MM, Romine LE, Lee YK, et al.. Spectral Doppler signature waveforms in ultrasonography: a review of normal and abnor-mal waveforms.Ultrasound .26(2):1997.83-99.

[6] Gielecki J, Zurada A, Sonpal N, et al. The clinical relevance of coeliac trunk variations. Folia Morphol .64(3):2005. 123-9.

[7] Oppo K, Leen E, Angerson WJ, et al). Doppler perfusion index: an interobserver and intraobserver reproducibility study. Radiol-ogy .208 (2):1998.453-7.

[8] Abu-Yousef MM. Normal and respiratory variations of the hepatic and portal venous duplex Doppler waveforms with simultaneous electrocardiographic correlation. J Ultrasound Med; 11(6):1998, 263-8. 
[9] Shapiro RS, Winsberg F, Maldjian C, et al. Variability of hepatic vein Doppler tracings in normal subjects. J Ultrasound Med.12 (12):1997. 701-3.

[10] Riahinezhad M, Rezaei M, Saneian H, Famouri F, Farghadani M..Doppler assessment of children with liver cirrhosis and portal hypertension in comparison with a healthy control group: An analytical cross-sectional study. J Res Med Sci. 30;2018.23:40

*Corresponding author.

E-mail address: abdelmoneim_a@ yahoo.com 